\title{
THE COLLIMATION OF MHD WINDS: BIPOLAR FLOWS AND JETS
}

\author{
Colin A. Norman \\ Department of Physics and Astronomy, \\ Johns Hopkins University and \\ Space Telescope Science Institute \\ and \\ Jean Heyvaerts \\ University of Paris
}

\begin{abstract}
We show that axisymmetric magnetized winds emanating from rotating stars, disks, tori, etc. generally tend to collimate along the symmetry axis and conjecture that this is the reason there are so many bipolar flows and jets. The actual form of the flows at large distances from the source are either cylindrical or paraboloidal depending on the total poloidal current.
\end{abstract}

\section{INTRODUCTION AND MOTIVATION}

There have been many detailed studies of the structure and the physics of magnetized winds including the solar wind (Weber and Davis 1967), stellar winds and their influence on magnetic braking (Mestel 1968), relativistic jets in the magnetospheres of black holes (Phinney 1983), bipolar flows and jets from young stellar objects (Pudritz and Norman 1986, Uchida and Shibata 1985) and the flows from compact relativistic stars such as pulsars (Goldreich and Julian 1970). The general approach taken in these papers is to assume the field line geometry and study the stationary behaviour of the conserved quantities such as the mass flux, the energy flux, the entropy flux and the angular momentum flux. The physics problem here is the analysis of the complex behaviour at the critical points (c.f. Kennel, Fujimura and Okamoto 1983).

As has been illustrated frequently at this conference, jets and bipolar flows are found to be associated with many astrophysical phenomena and the hypothesis that we propose here is that all jets and bipolar flows are the result of magnetized flows emanating from rotating, magnetized axisymmetric bodies. This hypothesis is consistent with all observations and relevant models.

The physics problem we are presented with is to analyse the shape of rotating axisymmetric MHD flows. Previous work on this subject can be summarized as follows. A comprehensive description of the equation for the general force balance across field lines for magnetized rotating flows with gravitational fields was developed by Okamoto (1975) and Heinemann and Olbert (1978). A similarity solution for magnetized flow off a disk was presented by Blandford and Payne (1982) for a specific current distribution on the disk found by making all the velocities equal to one another (the Alfvén, the Keplerian, and the sound velocity). Sakurai (1985, 198i) produced numerical solutions of the stationary partial differential equations and found 
collimation along the axis and supercomputing simulations are underway (Evans and Hawley 1988).

The approach I will outline here is to use asymptotic analysis and determine the shape of the field lines and the flow structure at large distances from the source subject to the boundary conditions, the critical points and the nonlinear interplay between the quantities conserved in the flow along the field lines. Many of the details behind the outline given here are to be found in the paper by Heyvaerts and Norman (1989; hereafter HN89).

\section{BASIC EQUATIONS}

The basic equations are a pair of partial differential equations namely the Bernoulli equation and the transfield equation.

The boundary conditions are given at the base of the field lines and are the angular velocity and the entropy at the base of each field line.

In these stationary axisymmetric flows there are critical points through which the flow must pass so that the flow is smooth and stationary. In this case these conditions determine the functional values of the specific angular momentum flow along a given field line, the specific energy flow along a given field line and the ratio of magnetic flux to mass flux along a given field line.

The pair of partial differential equations mentioned above are solved to produce the shape of the Alfvén surface and the density distribution at the Alfvén surface.

We emphasize that this analysis has no dead zones or closed field topologies, no allowance for reconnection, and is only applicable to stationary flow.

Further details of the equations mentioned here are found in HN89.

\section{ASYMPTOTIC FIELD LINE SHAPE}

It can be shown that the asymptotic equation for the shape of the field lines can be written in the form of the curvature of the field lines at large distances from the source. We know that the curvature must vanish asymptotically and consequently the expression for the curvature must be identically zero. This condition leads to an additional integral of motion that allows us to classify the different possible solutions for the asymptotic field lines. The physically relevant controlling parameter for the solutions is the total net current supplied at the source. If this current is finite the asymptotic shape of the field lines is cylindrical and if this curent is zero the asymptotic shape of the field lines is paraboloidal.

The paraboloidal asymptotics associated with zero poloidal current can be obtained by an asymptotic expansion in the $r^{-1}$, keeping all terms greater than $r^{-2}$ as $r$ tends to infinity. The zeroth order field line shape is

$$
r(a, z)=K(a) z^{p(a)}(\ln z)^{q(a)}
$$

and matching terms to order $(\ln z)^{-n}$ the form of the solution is

$$
r(a, z)=A \exp \left(\mu \int_{0}^{a} \frac{\Omega\left(a^{\prime}\right) d a^{\prime}}{\sqrt{E\left(a^{\prime}\right)}}\right) z^{\lambda \int_{0}^{a} \frac{\Omega\left(a^{\prime}\right) d a^{\prime}}{\sqrt{E\left(a^{\prime}\right)}}}
$$

where $\lambda$ and $\mu$ are constants along a field line and $\Omega(a)$ and $E(a)$ are the angular velocity and the energy flow on a field line. 
For cylindrical asymptotics the essential pair of partial differential equations simplifies and can actually be solved in quadrature as

$$
r_{\infty}(a)=\exp \left(\frac{2}{k \mu_{o}} \int_{o}^{a} \frac{\Omega(a) d a}{2(E-k \Omega / \alpha)^{\frac{1}{2}}}\right)
$$

where $r_{\infty}(a)$ is the cylindrical fieldline radius at infinity along field line a, $\alpha(a)$ is the ratio of the magnetic flux to mass flux along field line a, and $k$ is a constant.

In the case of no flow the basic equations reduce to the well known Grad-Shafranov equation.

One finds when analyzing the structure of these equations in detail that there is a complex interplay between the boundary conditions and the critical points. The critical points are the fast and slow magnetosonic points, and the Alfven regularity condition that there is no kink in the field lines at the Alfvén surface. It turns out that the Alfven critical point condition is redundant. The above asymptotic analysis is based on the assumption that there are no pathological interconnections between the physical quantities that result from these relationships. An understanding of these complex nonlinear boundary conditions is important for the simulations since even a simple change in boundary conditions from specifying mass flux rather than density and velocity at the base of a field line can potentially lead to problems.

\section{SUMMARY AND CONCLUSIONS}

The underlying physics of the collimation demonstrated in this paper is due to the increasing dominance of inertial forces as the flow pases beyond the Alfven surface. These inertial forces sweep the poloidal field lines back converting poloidal field to toroidal field. The poloidal field falls off with cylindrical radius as $r^{-2}$ but the toroidal field component falls off only as $r^{-1}$. When this dominates, the pinching effect of the hoop stress collimates the field.

One point to note about the similarity solutions for disks is that they may appear to violate the theorem given here since they are paraboloidal but do not appear to have zero total poloidal current. In fact they do agree precisely with the theorem since there is a singular return current along the axis that makes the total poloidal current zero consistent with paraboloidal asymptotics.

In summary, we have presented a general property of rotating, axisymmetric, magnetohydrodynamic winds with no closed field line topologies namely that these field lines collimate along the symmetry axis as cylinders and paraboloids if the total poloidal current is non-zero or zero respectively. This can account for the shaping of many astrophysical flows such as bipolar flows and jets associated with objects ranging from black holes to protostars.

\section{References}

Blandford, R. D., and Payne, D. G. 1982, M.N.R.A.S., 199, 88.

Evans, C. R., and Hawley, J. F. 1988, Ap. J., 332, 659.

Goldreich, P., and Julian, W. H. 1970, Ap. J., 160, 971.

Heinemann, M., and Olbert, S. 1978, J. Geophys. Res., 82, 23.

Heyvaerts, J., and Norman, C. 1989, Ap. J., in press.

Kennel, C. F., Fujimura, F. S., and Okamoto, I. 1983, Geophys. Ap. Fluid Dyn., 26, 147. 
Mestel, L. 1968, M.N.R.A.S., 138, 359.

Okamoto, I. 1975, M.N.R.A.S., 173, 357.

Phinney, E. S. 1983, Ph.D. thesis, University of Cambridge.

Pudritz, R. E., and Norman, C. A. 1986, Ap. J., 301, 571.

Sakurai, T. 1985, Astr. Ap., 152, 121. . 1987, Pub. Astr. Soc. Japan, 39, 821.

Uchida, Y., and Shibata, K. 1985, PASJ, 37, 515.

Weber, E. J., and Davis, L., Jr. 1967, Ap. J., 148, 217.

MORRIS: It should be stressed that there are several examples of bipolar nebulae surrounding evolved stars which show very high velocity polar outflows. The flows in these "preplanetary nebulae", as well as those in a number of planetaries, are presumably the result of magnetically-driven winds similar to those hypothesized for protostellar accretion disks. In the evolved star case, the accretion disk may form around a binary companion to the mass-losing red giant. So it is noteworthy that the magnetically-driven wind phenomenon can be found at both ends of stellar history.

KUNDT: Through what distances do you expect your jets to remain focussed? For jets consisting of stellar-wind material, my reservations are expressed in Astrophys. Space Sci. 148, 343 (1988). They relate to the huge required momentum transfer and to the mechanism by which narrow jets blow elongated cavities at high Mach numbers. 